\title{
Head-to-head comparison of myocardial perfusion SPECT and CMR for assessment of myocardial ischemia
}

\author{
Fredrik Hedeer*, Marcus Carlsson, Håkan Arheden, Henrik Engblom \\ From 18th Annual SCMR Scientific Sessions \\ Nice, France. 4-7 February 2015
}

\section{Background}

Myocardial perfusion single photon emission computed tomography (MPS) and cardiac magnetic resonance (CMR) can be used for assessment of myocardial ischemia. The methods reveal different aspects of the pathophysiology associated with myocardial ischemia where MPS is based on uptake of a perfusion tracer in viable mitochondria whereas CMR is based on firstpass perfusion reflecting coronary in-flow kinetics. The aim of this study was to perform a head-to-head comparison between MPS and CMR for assessment of myocardial ischemia under identical perfusion conditions at stress.

\section{Methods}

Ninety-six patients (34 females) were included in the study. All patients completed both gated MPS and CMR at rest and during adenosine stress. The MPS and CMR perfusion tracers were injected in the MR scanner during the same adenosine stress session. MPS images were acquired approximately one hour after the first-pass perfusion images. Qualitative image analysis for stress-induced ischemia was performed by two expert readers blinded to all clinical data.

\section{Results}

There were disagreements between MPS and CMR in 29\% (28/96) of the patients. In 17 patients CMR showed signs of stress-induced ischemia with a normal MPS and in 11 patients the MPS was positive with no signs of stress-induced ischemia with CMR (Table 1). Figure 1 shows an example of a patient where CMR shows stress-induced ischemia but no ischemia is seen with MPS. The Cohen's kappa value for the agreement between MPS and CMR regarding stress-induced ischemia was 0.15 .

\section{Conclusions}

When assessing myocardial ischemia in patients under identical myocardial perfusion conditions at stress there are disagreements between MPS and CMR in a significant number of cases. The prognostic significance of these findings remains to be determined.

\section{Funding}

N/A.

Published: 3 February 2015

Table 1 Number of patients with myocardial ischemia for MPS and CMR respectively

\begin{tabular}{lll}
\hline & MPS + & MPS - \\
\hline CMR + & $7(7 \%)$ & $17(18 \%)$ \\
\hline CMR - & $11(11 \%)$ & $61(64 \%)$ \\
\hline CMR, cardiac magnetic resonance MPS, myocardial perfusion SPECT; + $=$ ischemia - = no ischemia &
\end{tabular}

CMR, cardiac magnetic resonance; MPS, myocardial perfusion SPECT; + = ischemia; - = no ischemia. 


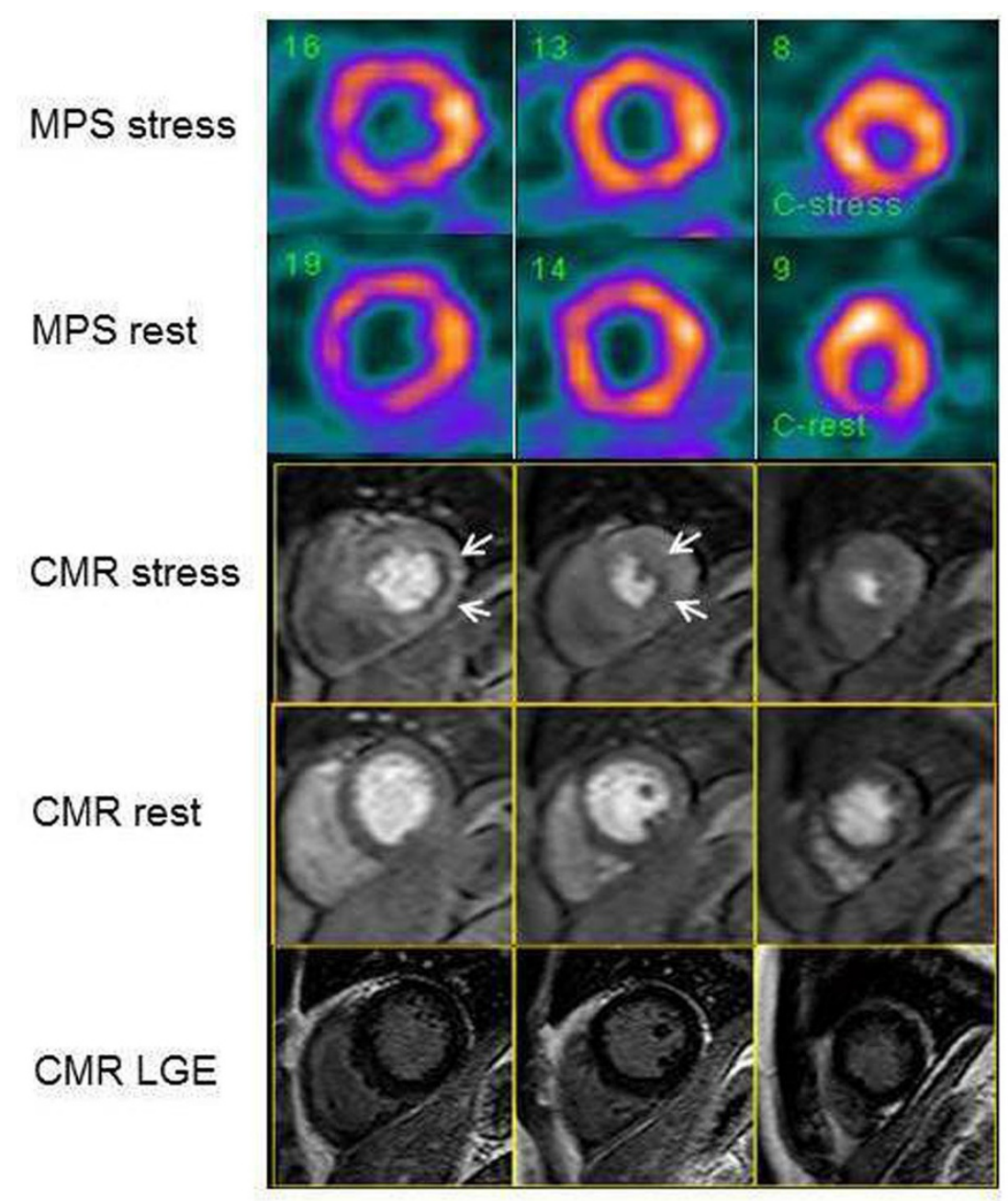

Figure 1 The figure shows short-axis slices of myocardial perfusion at rest and stress with myocardial perfusion SPECT (MPS) and first-pass perfusion at rest and stress with cardiac magnetic resonance (CMR). The MPS and CMR perfusion tracers were injected in the MR scanner during the same adenosine stress session. This is an example of a patient with no stress induced ischemia with MPS. CMR, however, shows stress induced ischemia in the subendocardial portion of the lateral wall (white arrows). CMR late gadolinium enhancement (LGE) images show no signs or myocardial infarction.

doi:10.1186/1532-429X-17-S1-P119

Cite this article as: Hedeer et al: Head-to-head comparison of

myocardial perfusion SPECT and CMR for assessment of myocardial ischemia. Journal of Cardiovascular Magnetic Resonance 2015 17(Suppl 1): P119.

\section{Submit your next manuscript to BioMed Central} and take full advantage of:

- Convenient online submission

- Thorough peer review

- No space constraints or color figure charges

- Immediate publication on acceptance

- Inclusion in PubMed, CAS, Scopus and Google Scholar

- Research which is freely available for redistribution 